\title{
Haemophilic Pseudotumor: Two Case Reports
}

Tan Kok Tong ${ }^{1^{*}}$ and Jameela Sathar ${ }^{2}$

${ }^{1}$ Master of Internal Medicine, National University of Malaysia, Malaysia

${ }^{2}$ Haematology, Hospital Ampang, Selangor, Malaysia

*Corresponding author: Tan Kok Tong, Master of Internal Medicine, National University of Malaysia, Malaysia, Tel: +60164199439; E-mail: tankoktong85@gmail.com Received Date: Aug 13, 2018; Accepted Date: Aug 20, 2018; Published Date: Aug 27, 2018

Copyright: (C) 2018 Tong TK, et al. This is an open-access article distributed under the terms of the Creative Commons Attribution License, which permits unrestricted use, distribution, and reproduction in any medium, provided the original author and source are credited.

\begin{abstract}
Haemophilic pseudotumor is a rare but chronic complication of hemophilia and it mimics osteosarcoma. The condition is related to the formation of encapsulated blood collection and necrosed tissues when the resorption mechanism of blood clot is compromised. Over years, the encapsulated lesion gradually enlarges and it erodes the surrounding tissues, including bones and joints. It is often diagnosed late due to its nature of slow progressive expanding haematoma. This condition is often found among patients with severe haemophilia who are poorly managed in their early years of life. We report two cases of haemophilic pseudotumor. Prophylactic factor VIII does not work in the first case and patient $A$ has to end up with operation to cure the enlarging haemophilic pseudotumor. The option of surgical intervention is emphasized for patient $A$ as he does not respond to treatment of prophylactic factor VIII. However, for the second case, the disease of patient B is under control with only prophylactic factor VIII and he does not require any surgical intervention and he is able to achieve regression of haemophilic pseudotumor. There are no established specific guidelines to approach this complication and thus the therapy is individualized with multidisciplinary approach involving haematologist, surgeon, pharmacist, physiotherapist, occupational therapist and dietician.
\end{abstract}

Keywords: Haemophilic pseudotumor; Haemophilia; Osteosarcoma; Haematoma

\section{Introduction}

Haemophilia $\mathrm{A}$ is a recessive $\mathrm{X}$-linked bleeding disorder which is caused by deficiency of factor VIII whereas haemophilia B, also named Christmas disease, is lacked of factor IX. One of serious complications due to haemophilia is the haemophilic pseudotumor and it is not commonly seen. As it is the mainstay of haemophilia in prevention of getting spontaneous bleeding, severe haemophilic cases are treated with prophylactic factor VIII. Haemophilic pseudotumor, although rare, is commonly found among patients with poorly treated with prophylactic factor VIII [1].

\section{Case Report}

We have two case scenarios of hemophilic pseudotumor. The first case was treated operatively. 55-year-old Chinese patient A was diagnosed with hemophilia $A$ at age of 10 years old when he sustained his left eye injury after fall. At age of 41 year old, he sustained right femoral fracture after fall and he underwent operation with fixation plate insertion. Further family history was obtained. His mother was a hemophilic carrier and his youngest uncle suffered from haemophilia. His two brothers succumbed to death due to severe bleeding (one brother had intracranial bleed and the other experienced massive bleeding post-cholecystectomy). One of his sisters was a haemophilic carrier. Since the time of diagnosis establishment till now, he had not been having regular follow-up till 2013, he presented to us (Hospital Ampang) with right thigh swelling with right knee pain over 6 months period. The swelling localizing at distal part of right thigh above knee was increasing in size gradually. Functionally, he had to ambulate with crutches since then. Clinically the distal part of right thigh was warm on touch and swollen with estimated size of $14 \times 20 \mathrm{~cm}$ above right knee. There were no bruishes or ecchymoses over the skin. Coagulation profile showed prolonged aPTT $(73.7 \mathrm{sec})$. Coagulation factor VIII activity was 3\% (normal ranges 50-200) with abscense of factor VIII inhibitor. Platelet count was normal. X-ray right lower limb showed intraosseous mass at right distal femur with intramedullary rod. The computer tomography of right lower limb showed that maximum cortical distruction was at the distal shaft of femur with the largest soft tissue mass measuring approximately $14.2 \mathrm{~cm} \mathrm{x} 14.4 \mathrm{~cm} \mathrm{x}$ $20.0 \mathrm{~cm}$ (Figure 1), predominantly hypodense $(20-70 \mathrm{HU})$ with patchy enhancement and specks of calcification; there was rim enhancement of the peripheral margins. Thus, haemophilic pseudotumor of right femur was diagnosed.

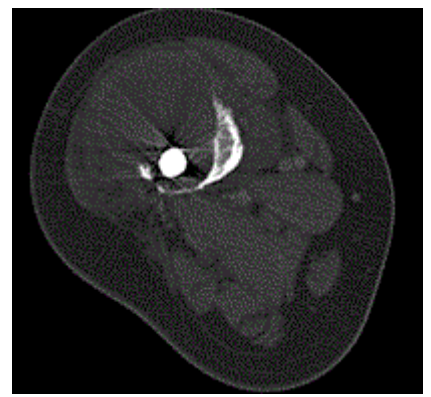

Figure 1: This feature is the image of MRI right femur (distal part) axial view with hetergenous lesion located at posterior compartment with size approximately $14.2 \mathrm{~cm} \times 14.4 \mathrm{~cm} \times 20.0 \mathrm{~cm}$.

Orthopaedic opion was sought and he was counseled for amputation. Initially he was not keen to undergo operation and 
Page 2 of 4

therefore managed with prophylactic factor VIII 10 units/kg 3x/week (Alleviate 3 vials, $3 \mathrm{x} /$ week<estimated $\mathrm{BW} \quad 60 \mathrm{~kg}$ without pseudotumor $>$ ). Nevertheless, in view of size increment of right leg haemophilic pseudotumor, he agreed to undergo operation of right hip disarticulation (Boyd procedure) in 2014. Now he is symptom-free with factor VIII prophylaxis (14 IU/kg 3x/week).
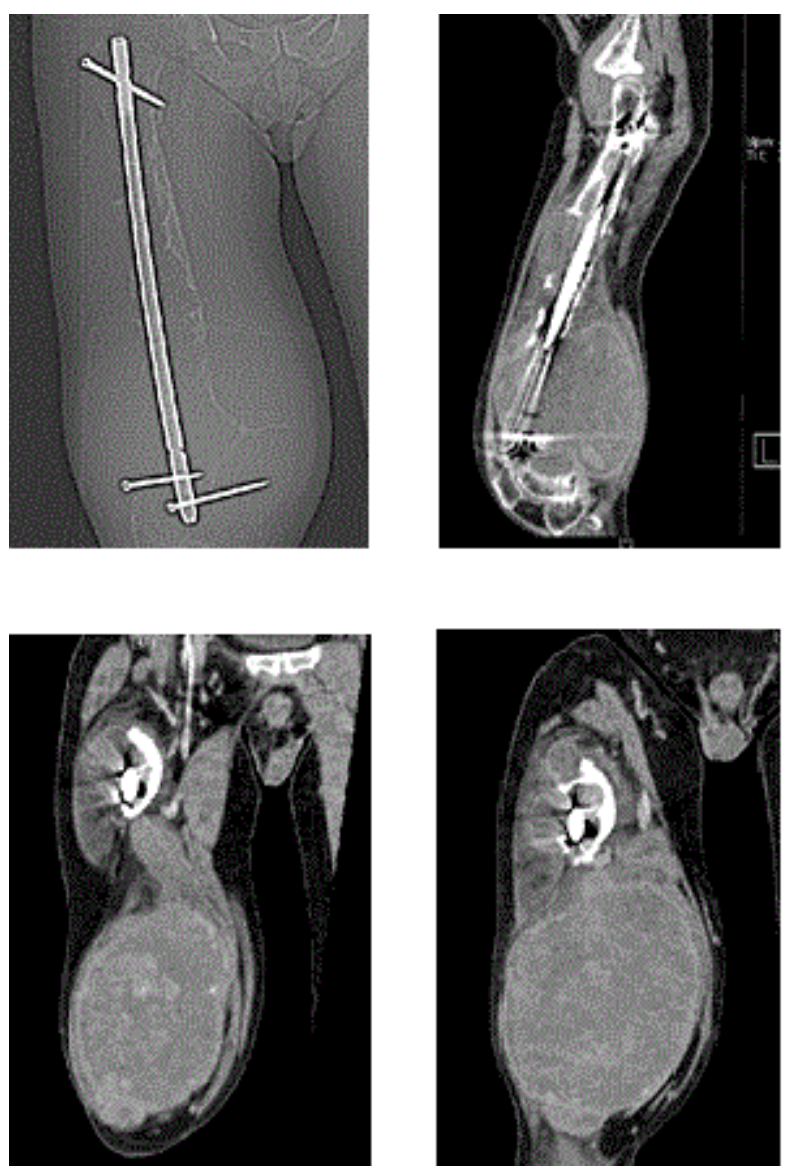

Figure 2: Images of (coronal view) MRI right femur. The entire shaft of femur (lesser trochanter to condyles) has central and eccentric expansile erosive lesions. Some of these are associated with soft tissue masses. There are soft tissue lesions within medulla of femur and surround the IMN. The most proximal lesion is approximately $1.5 \mathrm{~cm}$ from the proximal screw along the anterior cortex. There are 2 adjacent eccentric lesions with soft tissues masses in the anterior compartment (Figures 2 and 3). The maximal cortical distruction is at the fistal shaft of femur with the largest soft tissue mass measuring approximately $14.2 \mathrm{~cm} \mathrm{x} 14.4 \mathrm{~cm} \times 20 \mathrm{~cm}$. The main bulk of mass is in the posterior compartment. The soft tissue masses are similar in the character but vary in size. The lesions although predominantly hypodense $(2070 \mathrm{HU})$ are slightly heteregenous with patchy enhancement and specks of calcification. There is rim enhancement of peripheral margins. The overlying muscle bundles are stretched but otherwise intact. There are no inflammatory changes in the overlying soft tissues.

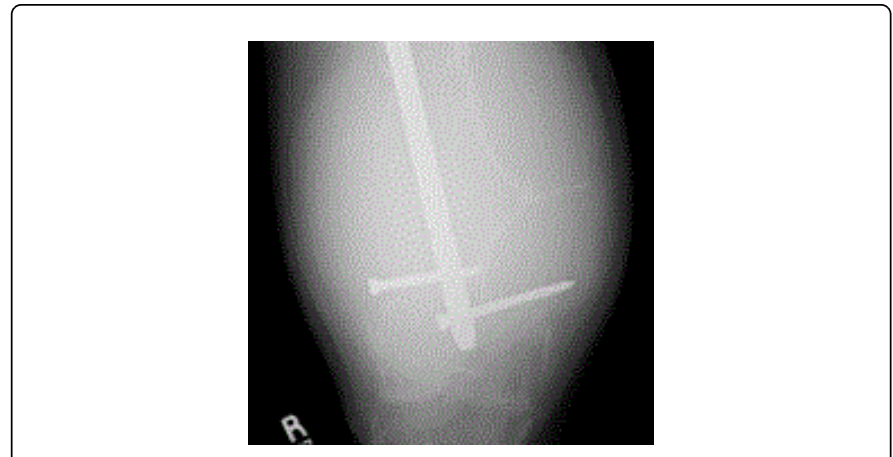

Figure 3: X-ray right femur (distally above knee) showing intraosseous lesion with calcified rim and intramedullary rod and nails (Taken on 21/2/13).

The second case of haemophilic pseudotumor was treated conservatively with factor VII level. 18-year-old patient B with background of severe Haemophilia A presented to us in 2013 with left knee swelling. He experienced intermittent pain and swelling within six months and the swelling subsided after monthly prophylactic factor VIII. He had neither fever nor any other bleeding tendency. He had been under care of other hospital but he sought second opinion at Hospital Ampang. Coagulation profile showed prolonged aPTT. Factor VIII activity was low (8.1\%) (normal ranging 50-200\%). Clinical examination of left knee was relatively normal. X-ray of left knee showed mass over left distal femur above knee (Figure 4). MRI of left lower limb showed heterogenous lesions $<5.5 \times 3.2 \mathrm{~cm}, 5.9 \times 1.3 \mathrm{~cm}$ (lateral) and $2.8 \times 1.2 \mathrm{~cm}$ (medial) $>$ involving the distal femur, extending into the knee joint and adjacent soft tissues (Figures 5 and 6 ); these findings were suggestive of left distal femoral haemophilic pseudotumor. We managed him with prophylactic factor VIII 3 vials $3 \mathrm{x} /$ week (BW-42 kg) and decided not to perform any invasive procedures. After $1 \frac{1 / 2}{2}$ years of pharmacological therapy (regular prophylactic factor VIII), the surveillance by using imaging tools showed that left distal femoral haemophilic pseudotumor has been resolving and reducing in size (Figures 7 and 8). Currently he is keeping well.

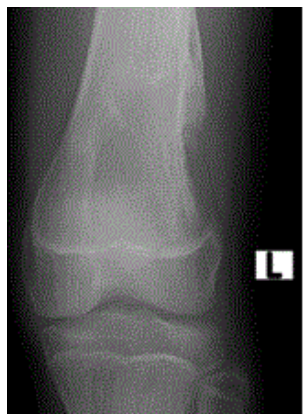

$6 / 6 / 13$

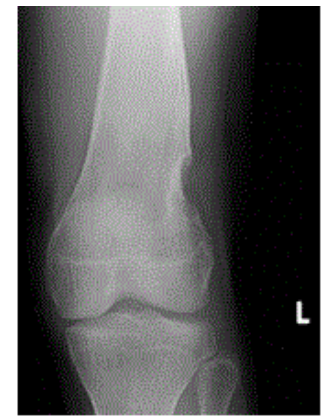

$15 / 12 / 14$
Figure 4: comparison of left knee x-ray before and after prophylactic factor VIII. Right picture showed swelling and osteolytic lesion over lateral aspect of left distal femur above knee. Left picture showed resolution of intraosseous lesion in comparing with right one. 
Page 3 of 4
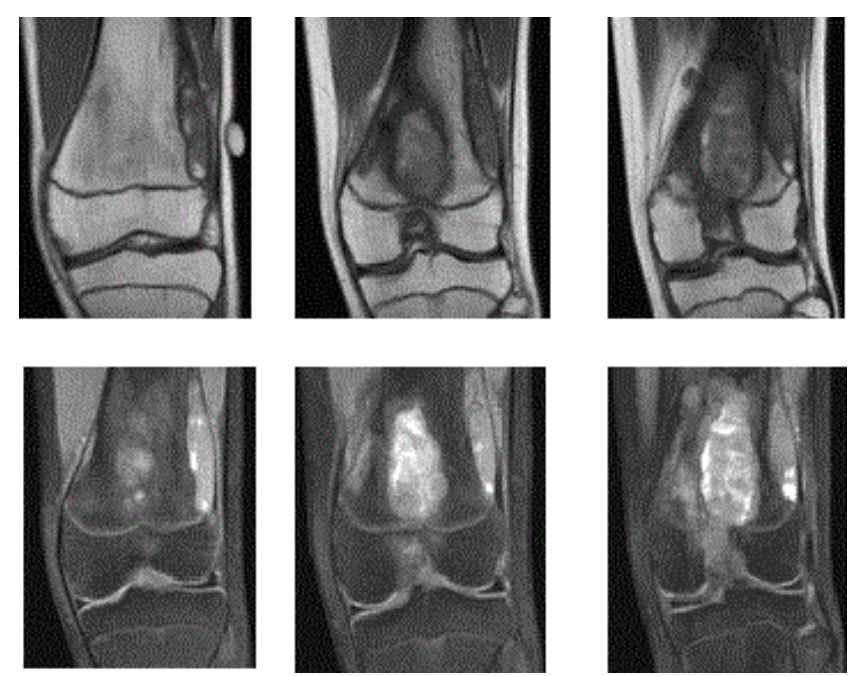

Figure 5: These images are sagittal view of left knee MRI (done on 7/6/13). Above three pictures showed T1 weighted and below ones showed T2 weighted. The MRI showed heterogenous lesions $<5.5 \mathrm{x}$ $3.2 \mathrm{~cm}, 5.9 \times 1.3 \mathrm{~cm}$ (lateral) and $2.8 \times 1.2 \mathrm{~cm}$ (medial) $>$ involving the distal femur, extending into the knee joint and adjacent soft tissues; these findings are suggestive of left distal femoral haemophilic pseudotumor.
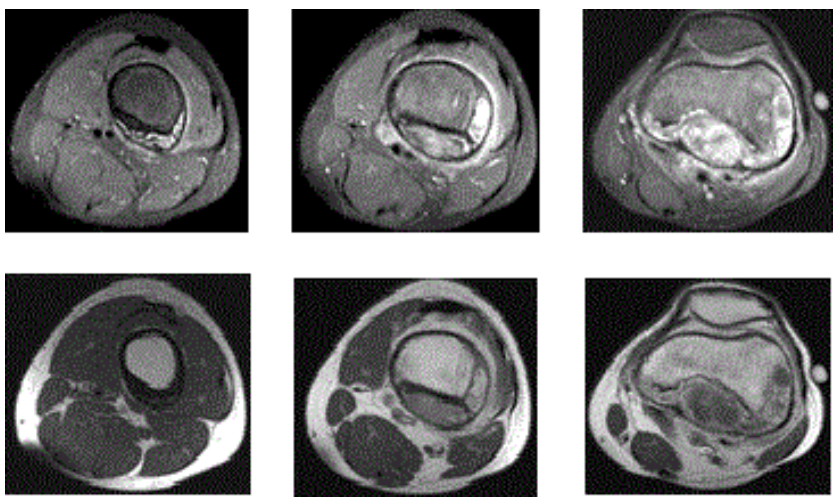

Figure 6: These images are axial view of left knee MRI. Above three images are Gadolinium-constrasted T1-weighted and below ones are T2-weighted. The MRI showed heterogenous lesions $<5.5 \times 3.2$ $\mathrm{cm}, 5.9 \times 1.3 \mathrm{~cm}$ (lateral) and $2.8 \times 1.2 \mathrm{~cm}$ (medial) $>$ involving the distal femur, extending into the knee joint and adjacent soft tissues; these findings are suggestive of left distal femoral haemophilic pseudotumor.

\section{Discussion}

Haemophilic pseudotumor is named so because clinically it mimics osteosarcoma. This diagnosis is first described by Fernandez Valderrama and Mathews in 1965 [2]. Among patients with severe haemophilia, $1-2 \%$ patients acquire haemophilic pseudotumor. Trauma is often related with the occurrence of haemophilic pseudotumor $[2,3]$. The condition is related to the formation of encapsulated blood collection and necrosed tissues when the resorption mechanism of blood clot is compromised. Over years, the encapsulated lesion gradually enlarges and it erodes the surrounding tissues, including bones and joints [4].

Haemophilic pseudotumor can be sub-grouped into proximal and distal type. In proximal type, the haematoma is formed either intramascularly or within soft tissue. Over long periods, it tends to invade adjacent structures, namely bone with subsequent mass effect. The distal lesion is frequently located at wrist, ankle, and skull; and the haematoma develops intraosseously [5-7]. In majority of patients, long bone is the common site of pseudotumor formation [8]. It is characterized by clinical physical findings of warm, tender and enlarging swelling of haemophilic pseudotumor and radiological findings of calcified cyst with hyper-opacity within. The diagnosis is established based on clinical examination as well as radiological investigations.
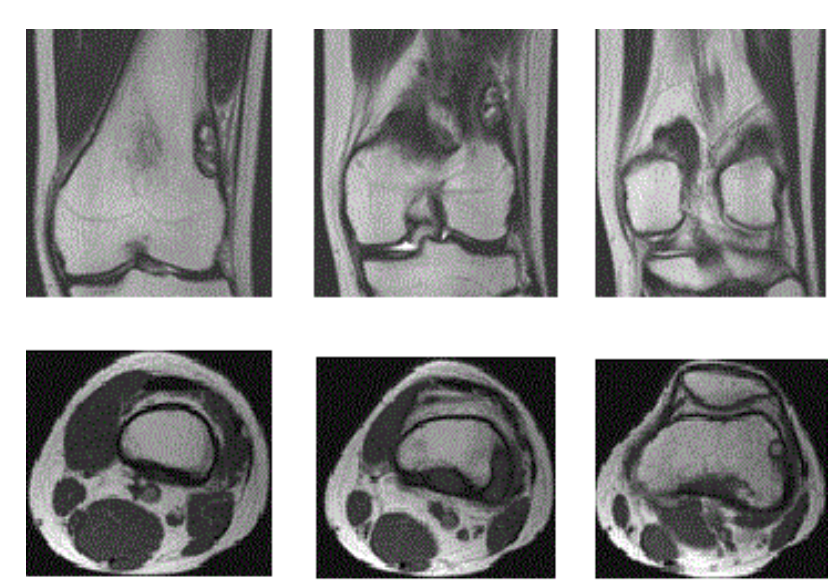

Figure 7: Above three images are sagittal views of T1-weighted MRI left knee (done in January 2015). Below three images are axial views of MRI left knee. These MRI images show resolution of haemophilic pseudotumor.
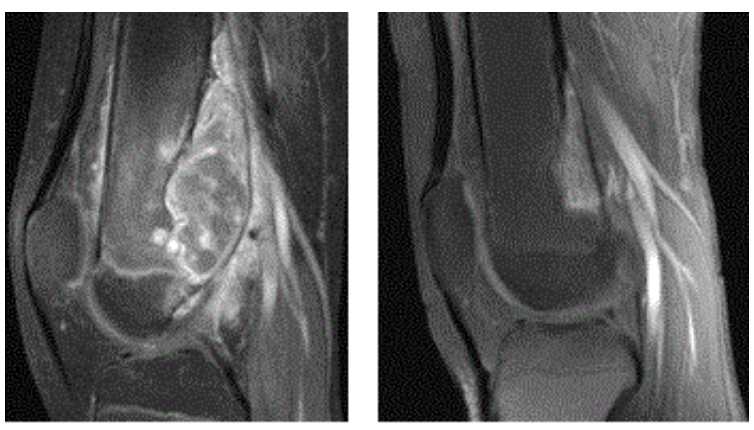

Figure 8: The Gadolinium-constrasted T1-weighted MRI image at the right side shows huge haemophilic pseudotumor (performed in 2013). In comparison with the MRI image at left, the haemophilic pseudotumor has regressed.

There are no established specific guidelines to approach this complication and thus the therapy is individualized [4]. The management of haemophilic pseudotumor involves multidisciplinary 
approach. Other than surgeons and haematologist, patients need care of physiotherapist, dietician, occupational therapist and pharmacist [9]. The indications of surgical interventions are the huge or enlarging haemophilic pseudotumor with mass effect and ineffective pharmacological therapy and presence of complications e.g. infected cyst or fistulae [5]. In the preparation for major operation to resect the pseudotumor, patients need administration of factor VIII. Radiotherapy and embolization could be considered in facing massive haemophilic pseudotumor [10]. Operation should be only undergone by experienced orthopaedic surgeon together with haematologists [11]. Pre-operatively and post-operatively, radiotherapy is alternative option to regress the haematoma size by inducing inflammation and fibrosis to the surrounding vessels [11]. Furthermore, radiotherapy could be opted to treat recurrent haemophilic pseudotumor of long bones [12].

Prophylactic factor VIII does not work in the first case and patient A has to end up with operation to cure the enlarging haemophilic pseudotumor. The option of surgical intervention is again offered for patient $A$ when he does not respond to treatment of prophylactic factor VIII. The operation is carried out in tertiary medical center with comanagement of haematologist and orthopaedic surgeon. However, for the second case, the disease of patient B is under control with only prophylactic factor VIII and he does not require any surgical intervention. Surgical resection in the second case can be optional in consideration of young age and small haemophilic tumor. He is able to achieve regression of haemophilic pseudotumor.

\section{Conclusion}

Haemophilic pseudotumor is the chronic complication of severe haemophilia and it is often diagnosed late due to its nature of slow progressive expanding haematoma. This condition is often found among patients with severe haemophilia who are poorly managed in their early years of life. Tiny haemophilic pseudotumor is firstly managed with prophylactic factor VIII. Surgical intervention would be the opted in cases with severe haemophilic pseudotumor or if the regression of lesion is not achieved with prophylactic factor VIII alone.

\section{References}

1. Jensen PS, Putman CE (1975) Hemophilic pseudotumor. Diagnosis, treatment, and complications. Am J Dis Child 129: 717-719.

2. Rodriguez Merchan EC (1995) The haemophilic pseudotumour. Int Orthop 19: 255-260.

3. Steel WM, Duthie RB, O'Connor BT (1969) Haemophilic cysts. Report of five cases. J Bone Joint Surg Br 51: 614-626.

4. Adams PT, Wharfe G (2013) A case of pseudotumour in a haemophiliac. West Indian Med J 62: 561-564.

5. Aneesh Pakala JT, Thomas J, Comp P (2012) Hemophilic Pseudotumor: A case report and review of literature. Int J Clin Med 3: 229-233.

6. Kumar R, Pruthi RK, Kobrinsky N, Shaughnessy WJ, McKusick MA, et al. (2011) Pelvic pseudotumor and pseudoaneurysm in a pediatric patient with moderate hemophilia B: Successful management with arterial embolization and surgical excision. Pediatr Blood Cancer 56: 484-487.

7. Heim M, Horoszowski H, Schulman S, Varon D, Barzilai A, et al. (1997) Multifocal pseudotumour in a single limb. Haemophilia 3: 50-53.

8. Rey EA, Puia S, Bianco RP, Pinto MT (2007) Haemophilic pseudotumour of the mandible: report of three cases. Int J Oral Maxillofac Surg 36: 552-555.

9. Valentino LA, Martinowitz U, Doolas A, Murali P (2006) Surgical excision of a giant pelvic pseudotumour in a patient with haemophilia A. Haemophilia 12: 541-544.

10. Ahuja SP, Sidonio R Jr, Raj AB, Bertolone SJ, Silverman C, et al. (2007) Successful combination therapy of a proximal haemophilic pseudotumour with surgery, radiation and embolization in a child with mild haemophilia A. Haemophilia 13: 209-212.

11. Panotopoulos J, Cihan AY, Klemens T, Funovics PT, Stockhammer V. et al. (2012) Surgical treatment of the haemophilic pseudotumour: A single centre experience. Int Orthop 36: 2157-2162.

12. Shah FY, Shah FY, Bhat S (2016) Hemophilic pseudotumors: Natural history and a case report of pseudotumor with femur fracture and swanneck deformity. Int J Orthop Sci 2: 10-14. 